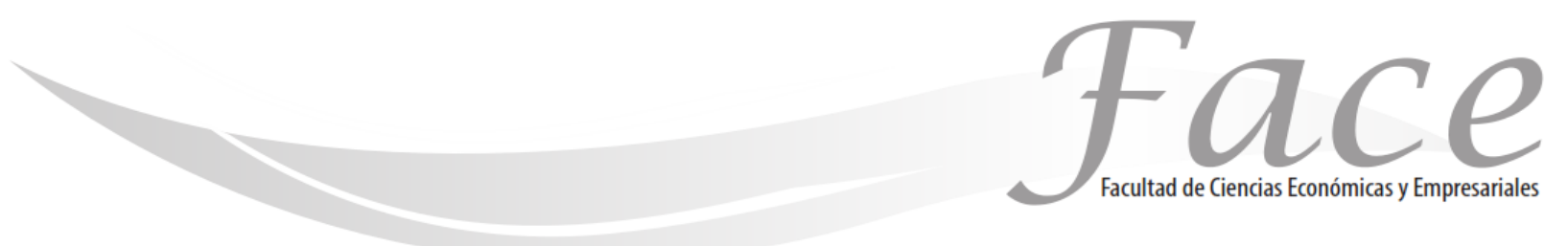

ISSN Impreso: 1794-9920

ISSN Electrónico: 2500-9338

Volumen 2-N¹

Año 2021

Págs. 5-18

(c) (i) ()

\title{
EL APORTE DE LA RESPONSABILIDAD EMPRESARIAL EN LA INDUSTRIA MANUFACTURERA DE BOGOTA, COLOMBIA
}

\author{
Diana María López Celis \\ Enlace ORCID: http://orcid.org/0000-0002-2208-9224 \\ Mónica Eugenia Peñalosa Otero \\ Enlace ORCID: http://orcid.org/0000-0002-7949-0625
}

Fecha de Recepción: Diciembre 2 de 2020

Fecha de Aprobación: Marzo 10 de 2021

\section{Resumen:}

A través de esta investigación, se exploró la percepción de las medianas y grandes empresas manufactureras de Bogotá acerca del aporte de los programas de responsabilidad social empresarial (RSE); a las ventas, imagen de marca, posicionamiento, sentido de pertenencia y exportaciones; siendo una investigación de alcance cualitativo, considerando el nivel de profundización y el target (personas encargadas de los programas de RSE en las empresas), se llevaron a cabo 9 entrevistas en profundidad apoyadas con técnicas proyectivas con una guía estructurada. La investigación permitió conocer que los programas de RSE en las medianas y grandes industrias manufactureras de Bogotá, aportan a la imagen y posicionamiento de marca al igual que a generar un mayor sentido de pertenencia por parte de los empleados, siendo relevante el papel de la RSE en las exportaciones; teniendo en cuenta que este es un factor importante tanto para las empresas como para el consumidor a nivel global, la cual abre puertas y representa una ventaja competitiva que contemplan las empresas al momento de realizar alianzas con empresas extranjeras.

Palabras clave: Ambiente, Actitud ambiental, Consumo Responsable.

Artículo de investigación resultado del proyecto titulado "Tipos de consumidores de acuerdo con la actitud ambiental: análisis comparativo entre San Luis De Potosí-México y Medellín-Colombia

*Profesora Asociada de la Facultad de Ciencias Económicas y Administrativas, Universidad Jorge Tadeo Lozano, Bogotá, Colombia. Magister en Psicología del Consumidor, especialista en Psicología del Consumidor. Profesional en Psicología. Investigadora Jr. de Colciencias. Se ha venido desempeñando en los últimos años como Docente - Investigadora en la Universidad Jorge Tadeo Lozano, la Universidad Central y la Universidad Javeriana- Colombia. Correo electrónico: diana.lopez@utadeo.edu.co

**Profesora Asociada de la Facultad de Ciencias Económicas y Administrativas, Universidad Jorge Tadeo Lozano, Bogotá, Colombia. Magister en Mercadeo Agroindustrial. Profesional en Relaciones Internacionales. Investigadora Jr. de Colciencias. Se ha venido desempeñando en los últimos años como Docente Investigadora en la Universidad Jorge Tadeo Lozano, la Universidad de La Salle y la Universidad de Pamplona - Colombia. Correo electrónico:

monica.penalosa@utadeo.edu.co 


\title{
THE CONTRIBUTION OF BUSINESS RESPONSIBILITY IN THE MANUFACTURING INDUSTRY OF BOGOTA, COLOMBIA
}

\begin{abstract}
:
Through this research, the perception of medium and large manufacturing companies in Bogotá about the contribution of corporate social responsibility (CSR) programs was explored; to sales, brand image, positioning, sense of belonging and exports; Being a qualitative research, considering the level of depth and the target (people in charge of CSR programs in companies), 9 in-depth interviews were carried out supported by projective techniques with a structured guide.

The research allowed to know that CSR programs in medium and large manufacturing industries in Bogotá, contribute to the image and brand positioning as well as to generate a greater sense of belonging on the part of employees, the role of CSR being relevant in exports; taking into account that this is an important factor for both companies and consumers at the global level, which opens doors and represents a competitive advantage that companies consider when making alliances with foreign companies.
\end{abstract}

Keywords: Corporate social responsibility, brand image, exports, sense of belonging, sales and positioning.

\section{A CONTRIBUIÇÃO DA RESPONSABILIDADE EMPRESARIAL NA INDÚSTRIA FABRICANTE DE BOGOTÁ, COLÔMBIA}

Resumo:

Por meio desta pesquisa, explorou-se a percepção de empresas manufatureiras de médio e grande porte em Bogotá sobre a contribuição dos programas de responsabilidade social corporativa (RSC); às vendas, imagem de marca, posicionamento, sentimento de pertença e exportação; Por ser uma pesquisa qualitativa, considerando o nível de profundidade e 0 alvo (responsáveis pelos programas de RSE nas empresas), foram realizadas 9 entrevistas em profundidade, apoiadas em técnicas projetivas com guia estruturado.

A pesquisa permitiu saber que os programas de RSE em médias e grandes indústrias manufatureiras em Bogotá, contribuem para a imagem e posicionamento da marca, bem como para gerar um maior sentimento de pertencimento por parte dos funcionários, sendo o papel da RSE relevante nas exportações; tendo em conta que este é um fator importante tanto para as empresas como para os consumidores a nível global, que abre portas e representa uma vantagem competitiva que as empresas consideram quando fazem alianças com empresas estrangeiras.

Palavras-chave: Responsabilidade social corporativa, imagem de marca, exportação, sentimento de pertencimento, vendas e posicionamento. 


\section{INTRODUCCIÓN:}

El sector empresarial tiene una importancia significativa para el desarrollo y crecimiento sustentable de cualquier país, en especial de aquellos en vía de desarrollo, por ser estas el motor de la economía. Ferraz y Ramos (2018). En la actualidad, el gran desafío para el desarrollo industrial y empresarial supone superar los retos en competitividad, de forma que permita al sector manufacturero específicamente acceder con éxito en las cadenas de valor y construir una base productiva de valor agregado diversificada.

Se conoce como sector manufacturero a las actividades que se dedican exclusivamente a transformar la materia prima a bienes de consumo. Estos se categorizan en dos clases de bienes: Los bienes intermedios que se utilizan como insumos para la producción de otros bienes, pasando a través de distribuidores que se encargan de aproximar el producto a sus diversos públicos de destino $\mathrm{y}$, los bienes finales que se consideran productos terminados así que no sufre una modificación para llegar al cliente final, por lo que no pasa por más intermediarios ya que está listo para su comercialización directa. La industria manufacturera es la producción en masa de estos productos, donde podemos encontrar a la agroindustria, textiles, fármacos, joyería, químicos, papel, equipos electrónicos, entre otros. Estas características lo convierten en el sector secundario de la economía ya que el primero es trabajar con la materia prima en bruto.
La Responsabilidad Social Corporativa (RSC) aplicada al sector empresarial está adquiriendo una gran importancia, debido a que genera influencia en la buena reputación de la empresa de cara a la sociedad y el sector manufacturero no es ajeno a esta realidad. La formación de una actitud ambiental, sustentada en las prácticas cotidianas, en la importancia que el individuo construye frente al tema ambiental y las relaciones con los productos que consume, entre otros. Es por esto por lo que este trabajo se enfocó en analizar el aporte de los programas de responsabilidad social empresarial (RSE); en este sector de la economía capitalina.

\section{MARCO TEÓRICO}

El entorno industrial en Colombia ha sido una temática extensa y con diferentes variables que han influido en su desarrollo. El sector manufacturero en Colombia ha sido líder en América Latina que ha progresado de manera más lenta que otros países, pero sin embargo ha evolucionado y ha sido partícipe del crecimiento en la economía del país.

La participación de la industria manufacturera en el PIB ha presentado una fuerte recesión desde la época de los 70's hasta llegar a inicios de los años 90's con pequeños crecimientos marcados por una industria más homogénea y un sector económico más sólido. Los buenos resultados de las grandes y medianas 
empresas han llevado a la ciudad a un crecimiento del $22 \%$ en el sector, del total de empresas registradas y renovadas, el $87 \%$ son microempresas (350.265), el $9 \%$ pequeñas empresas (38.151), el $3 \%$ medianas (10.533) y el $1 \%$ grandes empresas (3.597). (Revista dinero,2017).

Concentrando así el 10,5\%, el registro de nuevas empresas aumentó en los subsectores de elaboración de panela en un $43,1 \%$, elaboración de aceites y grasas de origen vegetal y animal con 29,0 \% y elaboración de alimentos preparados para animales con $16,1 \%$, la participación total de unidades productivas a nivel departamental se evidenció en Bogotá.

\section{Responsabilidad social empresarial (RSE)}

El origen etimológico de la palabra responsabilidad, deriva del verbo latino "respondere", que proviene del sufijo "abilis" y significa a la capacidad de desarrollarla. Por lo tanto, la palabra significa en su sentido más básico la capacidad de responder de manera adecuada a las obligaciones y expectativas de la vida que tiene la empresa. (Acevedo Guerrero. et al, 2013). En este mismo sentido Horacio Martínez (2011) resalta la implicación de la palabra responsabilidad haciendo referencia a: "Hacer bien y a tiempo lo que sabemos que corresponde sin que nadie nos lo ordene asumiendo las consecuencias, o sea, dar cuenta de las acciones realizadas" (pg. 109). Para Ojeda, (2019), la responsabilidad social es un tema que ha existido, en sus primeras fases, desde el principio de las civilizaciones, es posible rastrear escritos que hacen referencia a esas preocupaciones, como Los Gathas, de Zaratustra, o el código de Hammurabi, o la Ética a Nicómaco (Ojeda, 2015a). Las fases son posibles distinguirlas en función de la ética, el equilibrio social, el bien común, el altruismo y la filantropía (Ojeda, Vega, Vera, 2015).

Las variables que involucra el concepto de la RSE en su evolución son:

1. Respeto y garantía de los derechos humanos, como mínimo obligatorio aceptable.

2. Estándares laborales que garanticen el cumplimiento de todas las obligaciones con una parte interesada básica como son los empleados.

3. Gestión del impacto ambiental en las que la empresa, previene, mitiga y apoya planes generales de mejora de las condiciones ambientales.

4. Lucha contra la corrupción. Las empresas deben comprometerse con una relación y gestión transparentes con las autoridades locales, regionales y mundiales, así como eliminar toda práctica que tolere o promueva la corrupción pública o privada.

5. Análisis relacional que involucra el ámbito social donde opere la empresa con planes y acciones que propendan por la mejora $y$ desarrollo del ámbito social y cultural de las zonas de operación de la compañía. 


\section{La Responsabilidad Social Empresarial y la marca}

La sociedad hoy en día percibe la empresa como un ente productivo per se que tiene toda la capacidad y puede generar un cambio significativo; se ha dado una transición de pensamiento en este sentido ya que como enfatiza (Cortina, 1996) primero se percibía la empresa como un mero generador de riqueza, motivados únicamente por incrementar sus ventas y utilidad, y ahora la gran mayoría consideran algunas empresas como una organización socioeconómica que es responsable de manera ética y moral con la sociedad. Sumado a lo anterior y de acuerdo con Cavazos, Jiménez \& Santana (2018), para lograr la sostenibilidad, es necesario adoptar nuevas formas de producción.

\section{Posicionamiento y ventas}

Dentro del mundo empresarial se es consciente de que tomar la ética como filosofía organizacional genera un gran potencial en la creación de posicionamiento, rentabilidad y valor, creando un mayor vínculo emocional de valoración y admiración con sus consumidores para así facilitar su recordación, credibilidad e impacto a largo plazo, ya que se caracteriza por ser positivo y creciente. De igual forma, como Adela Cortina (2012) afirma en la inauguración del seminario ÉTNOR de Ética Económica y Empresarial:

"Las empresas deben dedicarse a contar historias que nos permitan entender los hechos, el cerebro humano es más un procesador de historias que un procesador lógico; en este sentido es fundamental que la empresa cuente buenos relatos para que la empresa se convierta en un referente de los ciudadanos".

\section{Fidelización de empleados (sentido de pertenencia)}

Al momento de llevar a cabo el desarrollo de la historia al público es imprescindible tener en cuenta el ambiente laboral, este es uno de los factores más importantes para la empresa, el trabajo óptimo y eficaz en equipo hacia un mismo objetivo, lo cual es una ventaja neurálgica sobre sus competidores, un gran atrayente al momento de la exportación e incrementa el valor de la empresa. Quienes se encargan de este proceso y del diseño de estrategias en la responsabilidad social empresarial son los stakeholders, ellos son "cualquier individuo o grupo que puede afectar o es afectado por el logro de los objetivos de la empresa" (Freeman, 1984); ellos saben que la metodología en la RSE al tener una naturaleza de reciprocidad es insaciable e insobornable, los consumidores son quienes analizan el efecto en ellos mismos y determinan el impacto e importancia en sus vidas. Los stakeholders por lo general sostienen dos principios, el primero consiste en que se debe involucrar a todo el personal con las reglas y el sistema corporativo; y el segundo trata de que la logística del personal por parte de la empresa se debe dar de manera incluyente e integradora, siempre teniendo en cuenta la ética, el desarrollo sostenible y el bien común. 


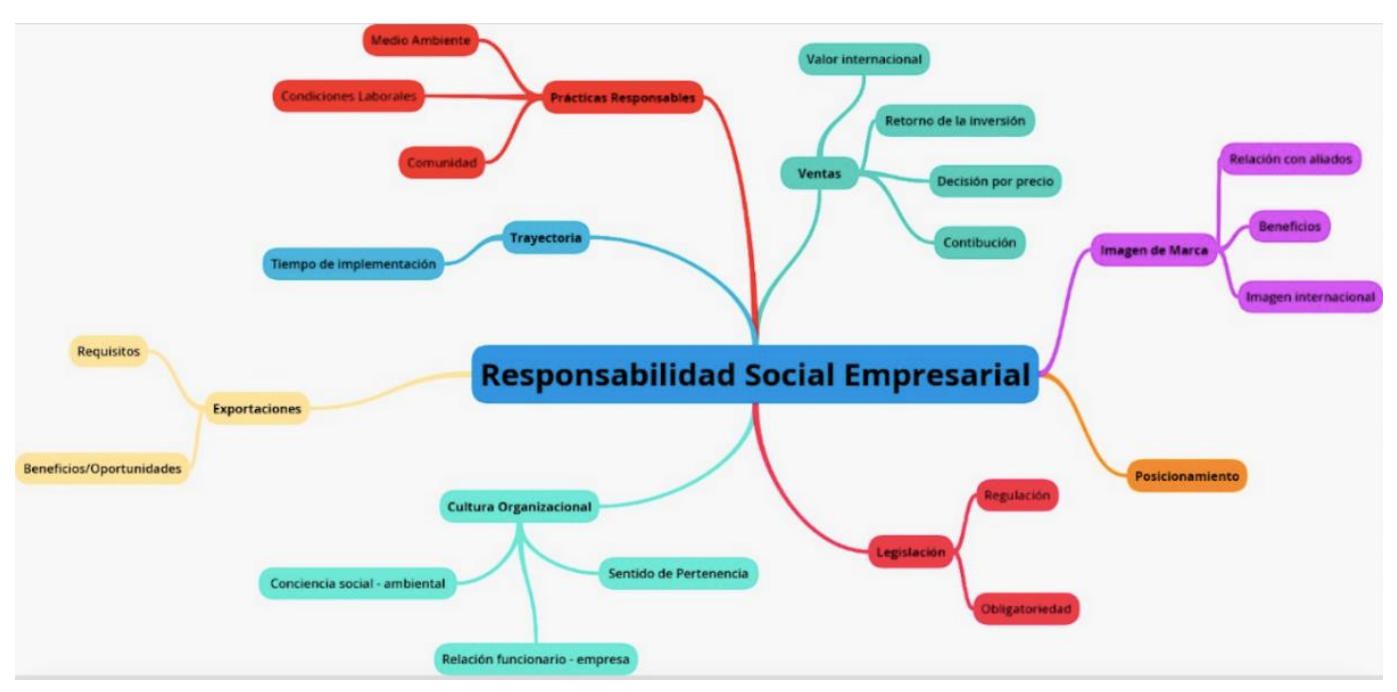

Fuente: elaboración propia: análisis realizado a través de Atlas ti 8.0

\section{Efecto de la RSE en las exportaciones y su contribución a la empresa}

La RSE termina siendo un imán para los mercados extranjeros, gracias a su propuesta diferencial de valor. Para reforzar la idea, tenemos que "los empresarios, diseñadores, proyectistas, etc, que sean capaces de catalizar tendencias sociales con intereses empresariales de modo que se aumente la calidad de vida laboral y que el respeto por el medio ambiente sea un aspecto más lucrativo, definitivamente están destinadas a triunfar, de eso no hay duda, y tendrá un éxito rotundo en el mercado doméstico e internacional" (Villa, 2007, p.55). De igual manera, así como lo afirma Camacho Parra, N., \& Soaza Forero, D. (2016). Los beneficios que genera ser una empresa socialmente responsable en Colombia (Tesis de maestría). Universidad de la Salle, Bogotá, Colombia. "Un gran atrayente para la inversión extranjera son los programas de Responsabilidad Social Empresarial, lo consumidores se fijan principalmente en quiénes realizan los productos, con qué materiales y con qué fin, son el principal atractivo".

\section{METODOLOGÍA}

El artículo cuenta con un enfoque exploratorio y alcance cualitativo. La técnica empleada para la recolección de información fue la entrevista en profundidad a través de la guía de entrevista en profundidad estructurada como instrumento. El target seleccionado fueron las empresas manufactureras medianas y grandes establecidas en Bogotá, que manejan programas de Responsabilidad Social Empresarial con más de dos años de implementación. La muestra fue de 9 empresas medianas y grandes del sector manufactura de Bogotá.

En cuanto a la unidad muestral se tomaron personas encargadas de los programas de responsabilidad 
social en las empresas manufactureras medianas y grandes en Bogotá, el método de muestreo fue por conveniencia y la fecha de trabajo de campo fue el segundo trimestre de 2019.

\section{RESULTADOS:}

El análisis fue realizado con la ayuda de la herramienta Atlas ti 8.0 estructurado, tomando como base las unidades o códigos, categorías y por último temas 0 familias, para la construcción de la red semántica.

El tema más destacado durante la investigación fueron las ventas, ya que un punto en común de todas las empresas entrevistadas fue que desarrollar el programa de Responsabilidad Social Empresarial es directamente proporcional a las ventas y ganancias de la empresa, así como lo afirma (Cortina, 2012) "la ética en una organización es rentable", esto debido a que es un gran diferenciador, logrando aumentar su competitividad y teniendo como resultado final en el consumidor una imagen positiva de marca y ubicarse ya sea en el top of mind y/o top of heart del cliente, así como lo enfatiza (Sallenave, 2004) "la capacidad de competencia de una empresa se basa en el aprovechamiento de una ventaja competitiva". (p.121).

A pesar de aumentar significativamente las ventas, la mayoría de las empresas reporta que estos resultados son más significativos en países extranjeros, ya que en el exterior se valora más a una empresa comprometida con el medio ambiente, con sus valores sociales y su trabajo con lo comunidad, por la confianza que estos le brindan al consumidor, y precisamente, esta es la decisiva al momento de efectuar la compra. Según el director general de Nielsen Iberia, Gustavo Núñez, "el consumidor español valora muy positivamente las marcas que incorporan atributos sociales 0 medioambientales y, como hemos visto, uno de cada tres está dispuesto a pagar más para incorporarlos a su cesta de la compra. Sin embargo, aún queda margen de mejora, tanto en disponibilidad de productos con sellos que certifiquen su compromiso social 0 medioambiental, como en el propio conocimiento de los certificados".

Sin embargo, en Colombia aún no se tiene el mismo impacto, las decisiones de los consumidores colombianos se caracterizan por su gran hipersensibilidad al precio sin olvidarse de la calidad, a pesar de que algunos estén dispuestos a recibir estímulos por los cuales pagarían a un precio más elevado.

El ambiente laboral es el factor más determinante para cualquier funcionario de una empresa, ya que este se convierte en su segundo hogar, el segundo lugar que más frecuenta y es necesario que disfrute lo que hace y en donde lo hace. Y por parte de la empresa este es el activo más valioso que ella posee, gracias a una eficiente planta de producción puede incrementar la productividad y la optimización de los resultados, satisfacer a los clientes, mejorar la comunicación y generar mayor motivación y seguridad; estos elementos dentro de este mundo globalizado en el que estamos resultan ser un gran atrayente que marca la diferencia con respecto a sus competidores e incrementa el valor de la empresa, Cristina Cortina afirma que (Cortina, 2012) "en una organización hay 
que tener como meta crear un clima ético, con unos valores que todas las personas compartan donde se toman decisiones y se actúa teniendo en cuenta estos valores" y finalmente eso es lo más importante, la relación entre el funcionario y el jefe o coordinador, evitar los largos procesos de comunicación, evitar las marcadas jerarquías, y que ellos noten que si la empresa crece, ellos también producen un ambiente más ameno y agradable para trabajar, así mismo incrementan su sentido de pertenencia y amor por la empresa, su fidelidad y compromiso, Cortina hace énfasis en este punto, "Si un trabajador está convencido que su empresa es buena y que le aporta valor luchará por conseguir los objetivos organizacionales y esto es precisamente lo que necesitan las empresas hoy en día que sus plantillas trabajen en equipo, unidas y en armonía."

El posicionamiento fue un tema clave en todo el desarrollo de la investigación, puesto que todas las empresas coincidieron en los impactos positivos que los temas de Responsabilidad Social han traído frente a la mejor percepción de los consumidores de la marca y la empresa y es que se está enfrentado diariamente a una sociedad más exigente y con mayores y más difíciles expectativas que suplir. "La sociedad hoy en día percibe la empresa como un ente que tiene toda la capacidad y puede generar un cambio significativo, primero se percibía la empresa como mero generador de riqueza, motivados únicamente por incrementar sus ventas y utilidad, y ahora la gran mayoría consideran algunas empresas como una organización socioeconómica que es responsable de manera ética y moral con la sociedad" (Cortina, 1996).
La sociedad necesita más empresas comprometidas con el medio ambiente, con las carencias de esta, con las necesidades que necesitan intervención, la sociedad busca en el poder empresarial formas de mitigar los problemas sociales y al poder percibir la ayuda de las marcas en todos los entornos sociales logra posicionarla mejor y hacerla cercana como una mejor y amigable empresa. Las compañías no son solo una imagen abstracta de un producto de consumismo, también existe un valor emocional que conecta a las personas, "Las empresas deben dedicarse a contar historias que nos permitan entender los hechos, el cerebro humano es más un procesador lógico, es fundamental que la empresa cuente buenos relatos"(Cortina, 2012); el posicionamiento corresponde a la imagen inmediata que se tiene de la marca, es un proceso cognitivo cargado de imágenes, situaciones y referentes positivos que logran cargar mentalmente a la persona y hacer de una marca algo más grande, es por eso que en el momento en que una empresa cuenta historias positivas de cómo ayuda a su entorno y lo hace mejor genera una consecuencia positiva en la mente de las personas. "La capacidad de competencia de una empresa se basa en el aprovechamiento de la ventaja competitiva" (Sallenave,2004), no solo se habla de ofrecer un producto o una experiencia de compra que satisface una necesidad, se puede ofrecer un valor agregado que conecte con el bienestar de las personas y esto puede reducirse a un diferencial en el mercado.

En cuanto a la imagen de marca las relaciones empresariales con aliados o los Stakeholders, parte desde el punto en que la empresa o marca es percibida, esto se da con intención pues la empresa 
genera estrategias que tienen como objetivo conseguir este tipo de imagen, una de estas estrategias son los proyectos de RSE que también llegan a este grupo de interés y que lo aceptan y muchas veces también aplican.

"La imagen de una empresa radica en su personalidad frente a sus clientes, proveedores, inversores, la prensa, el gobierno y el público en general" (Ramírez Sandoval \& Gochicoa Gramer, 2010). En el caso de las entrevistas que se realizaron a las empresas manufactureras, se puede resaltar la importancia de las relaciones que se establecen con los aliados 0 proveedores, no sólo es importante generar un impacto en los consumidores finales, sino también generar en toda su cadena de valor el mismo ADN o estrategia, para que así mismo se vea una unidad y coherencia.

Por otro lado, entre los beneficios que se pueden destacar además de los monetarios o los que se ven reflejados en las ventas, están los intangibles, la reputación, el valor emocional, la identificación de los consumidores, posicionamiento, entre muchos más. Para las empresas es claro que su actividad es un negocio, pero qué pasa si hacen parte de su negocio y de su cadena productiva todo lo que tiene que ver con proyectos de RSE, para así mismo minimizar costos y generar un impacto significativo en la comunidad en general (medio ambiente y consumidores). Según Ramírez \& Gochicoa (2010) la cadena de valor de una empresa frente a su competencia lo hace ser diferenciador y competitivo, se crea valor para los consumidores en el margen que es la diferencia entre el valor total y el costo colectivo que implica desempeñar las actividades de valor.
Al tocar el tema de la imagen de marca a nivel internacional, las empresas buscan explorar nuevos mercados y al tener proyectos de RSE les permite encontrar clientes diferentes a los que pueden encontrar en Colombia, los cuales les da un valor y un peso mayor a los proyectos de RSE y por lo tanto le apuestan a iniciativas sociales y medioambientales, queriendo que también hagan parte de su marca 0 empresa. Un factor importante para que esto suceda, es la manera en que las empresas logran comunicar los proyectos que realizan para que así mismo sus consumidores o clientes internacionales los apropien.

Las exportaciones en mercados maduros, los programas sólidos de RSE son un requisito informal que facilita la relación con las empresas de estos lugares, donde los estándares son un requisito informal pero cada vez mayor y más exigido por el consumidor final que pide compromiso no sólo de la empresa local sino de sus socios y miembros de la cadena de valor. En este contexto, el RSE es un requisito para entrar al mercado y facilita el acceso a las oportunidades. Este resultado es consistente con las conclusiones de las diferentes cumbres sobre RSE que llevaron al establecimiento de los principios del Pacto Global de la $\mathrm{ONU}$, las cuales fueron iniciativas principalmente impulsadas por países europeos, de la OCDE y otros países desarrollados, como reportaron tanto la Red Española del Pacto Global, como en la revisión histórica hecha por el investigador colombiano Ulises Tinoco Cantillo. 


\section{DISCUSIÓN Y CONCLUSIONES}

La investigación identificó que la condición actual de los programas de RSE en el nivel de norma técnica, es consistente con lo planteado en el marco teórico acerca de estos programas y las normativas a nivel internacional; este mismo reconocimiento to hacen todas las empresas encuestadas que tienen claro que no es un requisito legal pero si un elemento que representa su compromiso, su reputación y la imagen de su marca. Tal forma de actuación, la interpretan autores como Kirton y Trebilcok, resumidos por Angel Cabo, recogidos en el artículo de Acevedo Guerrero Et. al, que caracterizaron las soft laws o normas blandas, en oposición a las hard laws o derecho duro, que reconocen el nivel de norma técnica y legislación blanda para estas iniciativas.

La situación es diferente en el medio nacional y regional de América Latina, pues no hay acciones del estado visibles diferente a la aprobación de la norma técnica como se planteó en el marco teórico. No hay señas de política pública; incluso los proyectos de ley que algunos legisladores intentan impulsar no han pasado los debates, mostrando la poca acogida y cultura local hacia estos programas. Acevedo Guerrero Et. Al, documentaron el escaso desarrollo de la normativa y el fracaso en este avance de los proyectos de ley en el Congreso de Colombia. Por tanto en el nivel local, su impacto genera imagen de ciudadanía pero no una ventaja como en mercados más desarrollados.
Respecto al impacto de los programas de RSE con la competitividad exportadora de empresas multinacionales con sedes en varios países que exportan desde sus propias filiales a otros mercados manejados por sus sedes locales, no había una preocupación sustancial por el tema de RSE, lo que demuestra la necesidad que la presión nazca del consumidor final y que se transforme en una cultura de comprometerse, exigiendo sostenibilidad como requisito para comprar. Este hecho es reconocido por las autoridades mundiales que incluso impulsaron una cumbre, para buscar el camino de compromiso de las empresas multinacionales con la política social, habiendo ocurrido ya la cumbre de empresas que dio origen al Pacto Global. La principal de ellas es la "Declaración tripartita sobre las empresas multinacionales y la política social" liderada por la OIT. Este hecho muestra que estas corporaciones con control de mercados no tienen el mismo compromiso que las empresas competitivas.

Finalmente, se reconoce hacer los programas correctamente desde el comienzo para que puedan acreditarse y demuestren que se construye un negocio responsable con RSE en toda la cadena de valor. Estos resultados, tienen además sustento, no solo en el plano teórico, sino también en la realidad práctica como demuestran investigaciones tales como la de Zysman Neiman y Hermann Hrdlicka, que encontraron correlación positiva importante entre el compromiso ambiental y social y la competitividad exportadora en empresas brasileñas.

Por último en la parte de legislación lo que debe quedar claro en la RSE es que aunque el estado con 
Diana María López Celis - Mónica Eugenia Peñalosa Otero

el pasar del tiempo ha generado una serie de actos jurídicos que regulan o condicionan el actuar social responsable de las compañias, no es un tema de imposición, sino de autocrítica y sentido de responsabilidad; bien lo expresa Horacio Martínez (2011) al definir responsabilidad así: "Hacer bien y a tiempo lo que sabemos que corresponde sin que nadie nos lo ordene asumiendo las consecuencias, o sea, dar cuenta de las acciones realizadas" (p. 109). Se debe entonces inculcar que, aunque sea obligatorio el tener un programa de RSE en cada entidad, no lo son así las acciones que se realicen o desencadenan desde esta dependencia, la responsabilidad voluntaria: "En este nivel ya no se trata de conductas exigibles sino deseables, con las que la empresa se compromete con la sociedad: cooperación en causas caritativas, en el voluntariado para la comunidad...son elementos que se incorporan, lo denominamos acción social de la empresa". (Díaz, 2014, p.16).

Se debe concluir afirmando que la legislación frente a la RSE no consiste solo en hacer cumplir a las compañías con los propósitos específicos de cada norma, sino cada acto legislativo.

\section{REFERENCIAS:}

Andreu, A.; Fernández, D.L. (2011).- "De la RSC a la sostenibilidad corporativa: una evolución necesaria para la creación de valor". Harvard Deusto Business Review, Dicembre, pp.5-21

Activo Legal SAS. (01 de 06 de 2013). Fundamentos legales de la Responsabilidad Social Empresarial en Colombia. Disponible en

http://www.activolegal.com/web/index.php/no ticias/actualidad/504-responsabilidad-socialempresarial-rse-colombia-fundamentoslegales

Alejandra López Salazar; José Felipe Ojeda Hidalgo and Martha Ríos Manriquez, (2017), La responsabilidad social empresarial desde la percepción del capital humano. Estudio de un caso, Revista de Contabilidad Spanish Accounting Review, 20, (1), 36-46

Alpina. (2017). Responsabilidad Social en Alpina . Recuperado el 2018, de Alpina. Disponible en http://www.alpina.com.co/prensa/noticias/seg un-expertos-alpina-tiene-el-mejor-sistemade-gestion-de-responsabildiad-socialen.colombial

American Marketing Association. (1995). AMA. Recuperado el 2018, de Dictionary: https://www.ama.org/resources/Pages/Dictio nary.aspx?dLetter $=\mathrm{B}$

Cavazos Reyes, Ernesto, Jiménez Zarate Violeta \& Santana Esparza Gil. (2018). La innovación como estrategia de sostenibilidad y su efecto en el rendimiento de la industria azucarera en México. En Revista FACE Vol 18 (1). Disponible en http://revistas.unipamplona.edu.co/ojs_vicein ves/index.php/FACE/article/view/3174/1692

Calvo, D. C. (2004). LAS DIFERENTES TEORÍAS QUE SUSTENTAN LA RESPONSABILIDAD SOCIAL DE LA EMPRESA: ESTADO DE SITUACIÓN Y PROSPECTIVA. Obtenido de CYTA. Disponible en http://www.cyta.com.ar/biblioteca/bddoc/bdlib ros/334_calvo.pdf

Cajal, A. (n.f). Lifeder. Obtenido de Sectores Económicos de Colombia: Primario, Secundario y Terciario Disponible en https://www.lifeder.com/sectoreseconomicos-colombia/

Carroll, Archie. (1999). Corporate social responsibility: Evolution of a definitional construct. Business \& Society. 38. 268-295. 
Casa Luker. (2018). Fundación Luker - Casaluker. Disponible en http://www.casaluker.com/responsabilidadsocial/fundacion-luker/

Cámara de Comercio de Bogotá (2017). Balance de la economía de la región BogotáCundinamarca. Bogotá D.C.: Producción Editorial CCB.

CEPAL/Sociedad Alemana de Cooperación (GTZ). (11 de 2003 ). La Responsabilidad Social Corporativa en un Marco de Desarrollo Sostenible. Santiago de Chile, Chile.

Contraloría General de la Nación. (2010). Industrias Manufactureras. Obtenido de Contraloría. Disponible en http://www.contraloria.gob.pa/inec/archivos/p 4411C.pdf

Española, P. M. (15 de 04 de 2015). Pacto Mundial. Recuperado el 02 de 03 de 2018, de 10 Principios. Disponible en http://www.pactomundial.org/category/aprend izaje/10-principios/

Emprendepyme. (n.f). Emprendepyme. Obtenido de Tipos de Empresas. Disponible en https://www.emprendepyme.net/tipos-deempresas\#bloque-1

En Colombia. (n.f). Definición y Clasificación de las Empresas. Disponible en https://encolombia.com/economia/empresas/ definicionyclasificaciondelaempresa/

López Arceiz, F. J., \& Bellosta, A. J. (2017). Corporate Social Responsibility and good Corporate Governance practices in Spanish Ethical Mutual Funds: Analysis of investee companies. Innovar, 27(65), 139-151. https://doi.org/10.15446/innovar.v27n65.6506 8
Ferraz, Joao \& Ramos, Luma. (2018). Inclusión financiera para la inserción productiva de las empresas de menor tamaño en América L. 10.13140/RG.2.2.27183.59047.

FUNDES, IKEI, y Banco Interamericano de Desarrollo. (2005). Situación de la Responsabilidad Social Empresarial de la MiPyme: El caso Chileno. Disponible en http://www.prohumana.cl/documentos/guiars e.pdf

Garay. (1998). Colombia: Estructura industrial e internacionalización 1967 -1996.

López Pineda, Luis Fernando. (2010). TRANSFORMACIÓN PRODUCTIVA DE LA INDUSTRIA EN COLOMBIA Y SUS REGIONES DESPUÉS DE LA APERTURA ECONÓMICA. Cuadernos de Economía, 29(53), 239-286. Retrieved February 09, 2021, from http://www.scielo.org.co/scielo.php?script=sci _arttext\&pid=S0121$7722010000200009 \&$ Ing=en\&tlng=es.

Herrera, H. M. (2011). Responsabilidad Social y Ética Empresarial . Bogotá: ECOE Ediciones.

Huesca, C. R. (2012). Comercio Internacional . Tlalnepantla, México: Red de Tercer Milenio S.C.

ICONTEC. (2008). Guía Técnica Colombiana, Responsabilidad Social. Bogotá, Colombia.

Maldonado, A. (2010). La evolución del Crecimiento industrial y transformación productiva en Colombia 1970-2005. Patrones y determinantes. Universidad Nacional de Colombia , Bogotá.

Malhotra, N. (2008). Investigación de Mercados . México : Pearson Educación.

Montoya, B \& Martínez Ramos, P. (2012) Responsabilidad Social Empresarial: Una respuesta ética ante los desafíos globales. Disponible en http://www.kas.de/wf/doc/kas_37565-1522-430.pdf?140425034037 
N. Rebolledo, C. Duque, L. Angel, \& A. Velasco. (2013). Perfil del Sector Manufacturero Colombiano. Universidad de Santiago de Cali, Cali.

Ojeda Hidalgo, José Felipe (2019). Responsabilidad social en instituciones de educación: propuesta de medición. En Revista FACE Vol 19 (1). Disponible en http://revistas.unipamplona.edu.co/ojs_vicein ves/index.php/FACE/article/view/3698/2094

Organización de las Naciones Unidas. (15 de 06 de 1999). ONU . Obtenido de 10 Principios Disponible en http://www.pactomundial.org/category/aprend izaje/10-principios/

Orozco Toro, Jaime Alberto; Roca Correa, David Construcción de imagen de marca y reputación a través de campanas publicitarias de RSC Sphera Pública, núm. 11, 2011, pp. 273-289 Universidad Católica San Antonio de Murcia Murcia, España

Peñaranda-Peñaranda, M. M., Rodríguez-Castilla, M. M., \& Soto-Arévalo, F. S. (2016). Responsabilidad social empresarial: una estrategia para conseguir las mejores prácticas administrativas. Revista Ingenio, 8(1),

115-122. https://doi.org/10.22463/2011642X.2056v

Peñalosa Otero, Mónica Eugenia Peñalosa, López Celis, Diana María \& Martínez Aguilar, Marco Francisco. (2020). Las actitudes medioambientales de los consumidores: análisis comparativo en las ciudades de San Luis de Potosí, México y Medellín, Colombia. En Revista FACE Vol 20 (1). Disponible en http://revistas.unipamplona.edu.co/ojs_vicein ves/index.php/FACE/article/view/4218/2549
Ramírez, \& Núñez. (1999). Estudio comparativo sobre el éxito exportador PYME en Argentina, Chile y Colombia. Disponible en https://books.google.com.co/books?id=vme3 30fWBnYC\&pg=PA16\&lpg=PA16\&dq=ramire $z+y+n u n ̃ e z+1999 \&$ source $=b \mid \& o t s=V t C 68 s B A$ ZS\&sig=NDcSZ3o8X30HhRO3OXJTOQqUd Qk\&hl=en\&sa=X\&ved=0ahUKEwi8g8zNnZAhUHsFMKHXjJDy0Q6AEINZAG\#v=0 nepage\&q=ramirez $\% 20 y \% 20$ nuñez $\% 201999$ $\& f=f a l s e$.

Ramírez Sandoval, J., \& Gochicoa Gramer, E. (2010). Imagen corporativa: ventaja competitiva para las organizaciones PYME . México.

Regalado, O. Allpacca, R. Baca,L.Gerónimo,M (2011) Endomarketing: estrategias de relación con el cliente interno Disponible en https://www.esan.edu.pe/publicaciones/2011/ 08/05/endomarketing_para_web_con_sello.p $\mathrm{df}$

Revista Dinero. (2017). "Exportaciones en Agosto apenas levantaron cabeza: Subieron 1,48\% frente a 2016". Obtenido de Comercio. Disponible en http://www.dinero.com/economia/articulo/exp ortaciones-en-agosto-de-2017-dane/250601

Saumitra N Bhaduri; Ekta Selarka. (2016) \& S. E, Corporate Governance and Corporate Social Responsibility of Indian Companies. Singapure: Sustainability, Ethics \& Governance.

Salcedo Díaz, Laura, Porto Solano, Andrés Felipe, Echeverry Gutiérrez, Camilo, Boss Agudelo, Jovanny, \& Moreno Ortiz, Carlos Alberto. (2016). Responsabilidad Social Empresarial: Modelo de procesos de desarrollo de productos con base en la Metodología PRiSM y la Estrategia P5. Producción + Limpia, 11(2), $111-$ 125. https://dx.doi.org/10.22507/pml.v11n2a1 0

Sánchez Sánchez, E., S.P, T. D., J.S, T. R., \& Z.M, P. F. (2011). Responsabilidad Social como éxito 
e innovación empresarial . Bogotá, Colombia: Universidad de Bogotá Jorge Tadeo Lozano.

Secretaria General. (1997). Sentencia T-375/97. Corte Constitucional. Bogotá: Relatoría.

Soto, B. (2010). Las ventajas de la responsabilidad social empresarial para la empresa. Disponible en https://www.gestion.org/lasventajas-de-la-responsabilidad-socialempresarial-para-la-empresa/

Tinoco Cantillo, U. (2013). Evolución, aproximación al concepto. Bogotá. Universidad Nacional de Colombia.

Vicaría, M. P. (0 de 01 de 2009). "SER O APARENTAR LA RESPONSABILIDAD SOCIAL EMPRESARIAL EN COLOMBIA: Análisis organizacional basado en los lineamientos de la Responsabilidad Social Empresarial". Disponible en http://www.javeriana.edu.co/biblos/tesis/com unicacion/tesis250.pdf 